\title{
Status of IR Array Technology for Wide-Field NIR Survey Cameras
}

\author{
Klaus W. Hodapp and Donald N. B. Hall \\ Institute for Astronomy, 640 N. A'ohoku Place, Hilo, HI 96720, USA
}

\begin{abstract}
Over the past two decades, infrared detector arrays have evolved from small, highly experimental devices to large format arrays being used in numerous facility class instruments. The next generation of infrared arrays will allow the design of mosaic instruments with very large fields of view that offer exciting new opportunities for large scale surveys in the infrared.
\end{abstract}

\section{Introduction}

This paper is intended to give a brief overview over the history and basic technology of infrared detector arrays, and to describe the current state the art of infrared detector arrays. Of particular interest in the context of observations of brown dwarves are the new opportunities for wide-field infrared surveys with the coming generation of large mosaic infrared cameras.

Infrared radiation was discovered in 1800 by Sir William Herschel, using conventional thermometers as rudimentary bolometers. The astronomical utilization of this part of the spectrum developed only slowly, limited by the availability of sensitive detectors. In the 1960's and through the 1970's, single detector elements came into widespread use, first $\mathrm{PbS}$ and later $\mathrm{InSb}$, largely for aperture photometry, but also for the first, rather crude 2-dimensional "maps" .

\section{Basic Technology of Infrared Arrays}

Infrared detector arrays combine two functions: Detection of infrared photons and storage and processing of the integrated signal. Early ideas of designing the readout electronics directly into the infrared material encountered overwhelming practical difficulties. The universally adopted solution, therefore, is a hybrid approach. The infrared photons are absorbed in a narrow-bandgap semiconductor material, and the resulting charge carriers separated in the electric field of a PN-junction or, for long-wavelength material, an externally applied electric field. One side of the PN junction is grounded, the other is connected to the read-out integrated circuit (ROIC), also simply called the multiplexer, via Indium bumps. Signal storage, the switching of the signal to an output amplifier, and transmission to external signal-processing electronics are the functions of the multiplexer, which is fabricated using well established silicon fabrication technology. The two components are connected via Indium columns grown by vapor deposition on both sides and then pressed together to form the electri- 
cal connection. The resulting hybrid device, often call sensor chip assembly (SCA), consists of materials with different thermal expansion coefficients and is subject to severe mechanical stresses when cooled to the cryogenic operating temperatures. The manufacturers have engineered several solutions to manage the mechanical stresses and reliable hybrid devices up to $38 \mathrm{~mm}$ x $38 \mathrm{~mm}$ (the HAWAII-2) have been produced.

Some of the earlier multiplexers for infrared arrays were based on the CCD design that is being used so successfully for optical imagers. However, the hybrid structure of infrared arrays does not allow the multiplexer to be built as a burried-channel CCD, instead the charge has to be transported through the surface of the silicon crystal, resulting in relatively high levels of noise. All large format infrared arrays for astronomy now use basically the same multiplexer design based on CMOS switched FET technology.

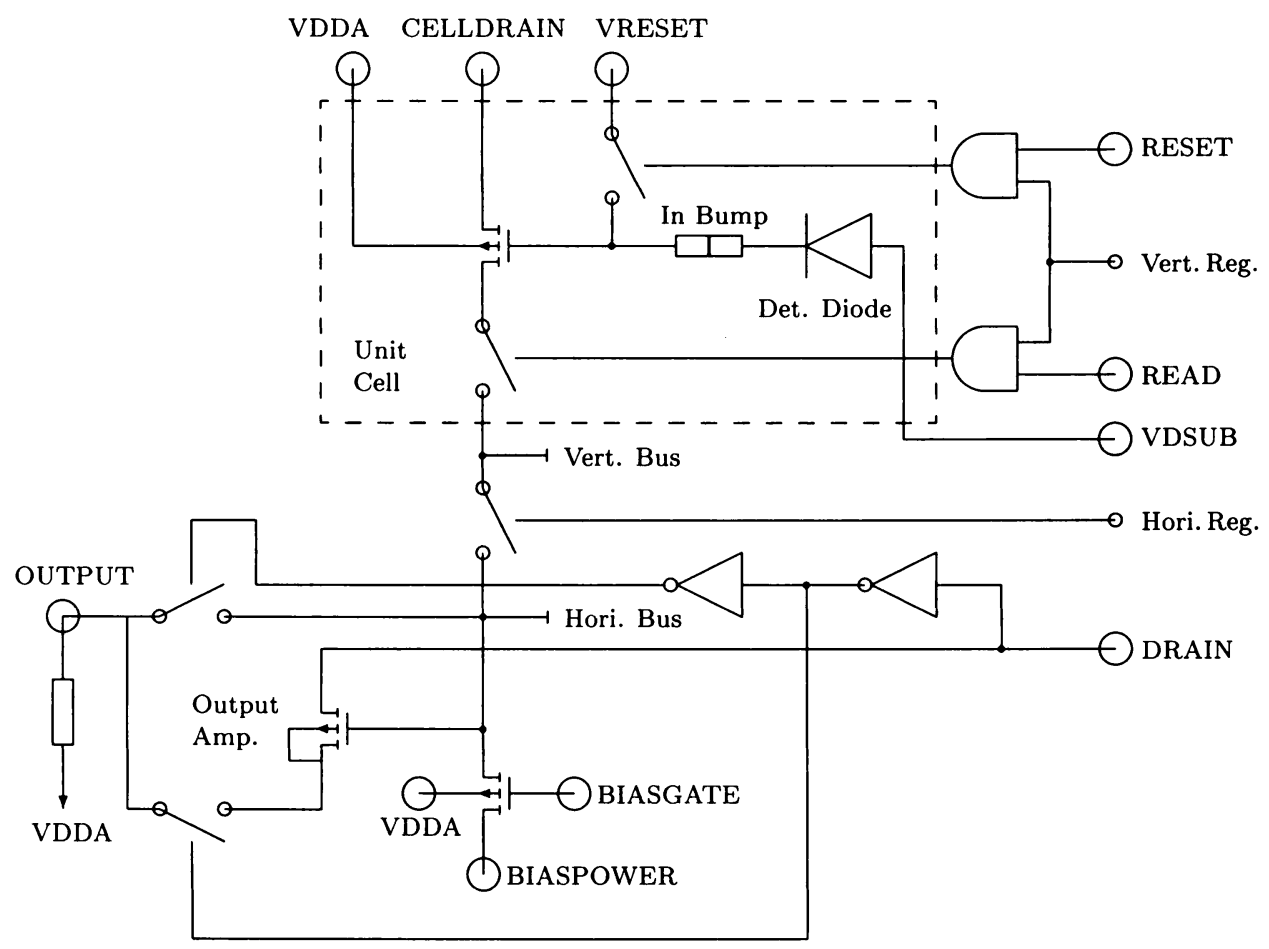

Fig. 1: Schematic Diagram of the HAWAII-2 Multiplexer

An individual pixel contains a minimum of 3 FETs, two of which are shown as switches in Fig. 1. The infrared sensitive diode is connected, via indium bumps, to the integrating node. An integration is prepared by switching the reset switch conductive and thereby connecting the diode to a reset voltage (VRESET) and charging the PN junction in back-biased (non-conducting) direction up to 
a typically $0.5 \mathrm{~V}$. After switching the reset FET into its non-conducting state, the charge left on the PN junction is now isolated: The reset FET has minimal leakage, there is minimal leakage from the gate of the charge-sensing FET to its source and drain, and the PN junction is biased in reverse direction. The only way for the unit cell to be discharged is through the generation of charge carriers in the PN junction by absorption of photons or by dark current. The measurement of radiation being absorbed by the infrared detector material is therefore done by observing the discharge of the unit cell. The charge remaining in the unit cell can be measured by feeding a current through the charge-sensing FET. This current goes from BIASPOWER to CELLDRAIN and is regulated by BIASGATE. It is directed to an individual unit cell by a system of column and row switches. The voltage drop across the source-drain of this FET is dependent on the voltage at its gate, i.e., on the charge remaining on the integrating node. This voltage drop can be measured at low impedance, and, if necessary, further amplified by an output FET. The charge remaining on the integrating node is not consumed or altered by the readout process, opening the possibility of reading the charge repeatedly to lower noise associated with the readout process.

\section{Readout Modes of Infrared Arrays}

This non-destructive nature of the readout of an infrared array is being used in a variety of readout schemes. The simplest possible readout mode, a reset of the device followed by a staring integration and one read at the end has the advantage of minimum overhead and is the best readout in situations of very large background, where readout speed is at a premium and read-noise is not an important contribution to the overall noise level.

When read-noise is important, this simple scheme suffers from a fundamental problem. Each time the device is reset, the reset level may vary due to instabilities of the reset voltage, but more fundamentally, the reset level is subject to kTC noise. This noise is the fundamental uncertainty in the amount of charge deposited on a capacitance connected to a voltage source. So in repeated reset-read integrations, the starting point is always uncertain within kTC (about 50 electrons under typical conditions). Once the reset switch isolated the charge stored in the unit cell, the charge is, of course, constant and can be measured by a first read of the device, prior to the start of the science integration. The difference between the read at the end of the integration and the one at the beginning is proportional to the integrated signal, and is obviously immune to shifts in the amount of charge initially deposited in the unit cell. This readout scheme is called correlated double sampling (CDS) and is the most commonly used readout scheme.

Read-noise can be further reduced by not only reading the device once at the beginning and end of the integration, but many times, thereby reducing the higher frequency components of the read-noise. This readout method is commonly known as Fowler sampling. While clearly advantageous in low background situations, its disadvantages are the extra time spent in reading the device out, thereby reducing efficiency. For multiplexers that emit photons during readout (electroluminescence or "glow"), each read contributes additional photons, until the shot noise of those photons dominates the read-noise. 
Efficiency is increased by the sample-up-the-ramp method, where reads of the arrays are spread over the entire integration time, and the signal is the slope of a least-square fitted line through the data points. Additional advantages of this method are that saturating pixels can be detected and the signal can be recovered, and that cosmic ray hits during the integration can be identified and recovered from.

\section{The Early Infrared Arrays}

The first infrared arrays became available to astronomers in the early 1980 s, having been developed originally for military purposes. The first device to be used in astronomical facility instruments was the Santa Barbara Research Center (SBRC) $58 \times 62 \mathrm{InSb}$ device, developed under contract from NOAO and ROE, soon followed by HgCdTe devices from Rockwell in $64 \times 64$ and $128 \times 128$ format developed for various JPL programs.

The competition for the infrared instrument for the Hubble Space Telescope (HST) motivated the competing manufacturers to develop low-noise and low dark current devices. The winner of this competition, the NICMOS3 device from Rockwell, became the most widely used infrared array for the first half of the 1990s, truly bringing infrared array technology into the mainstream of astronomical instrumentation. These were the devices used in the 2MASS sky survey, and some are still in use today. Shortly after the introduction of the NICMOS3, the Santa Barbara Research Center (SBRC) introduced a $256 \times 256$ $\mathrm{InSb}$ detector array that was used in all of the instruments requiring sensitivity out to $5.5 \mu \mathrm{m}$.

\section{The Large Format Arrays}

After completing the development phase for NICMOS, there was no further funding from NASA for large-format near-infrared detector arrays until the very end of the decade. The step to $1024 \times 1024$ Format was taken by ground-based observatories, with all or part of the funding from military sources. The HAWAII devices (now called HAWAII-1 devices to distinguish them from later versions) were developed by Rockwell under contract from the IfA, with funding provided by the Air Force Research Lab for the AEOS telescope spectrograph project (Hodapp et al. 1996). The Aladdin project at SBRC to develop a $1024 \times 1024$ InSb device was similarly funded by NOAO in collaboration with the U.S. Naval Observatory (Hoffman et al. 1998).

In 1998 , another major step in detector array size to $2048 \times 2048$ format was taken with the HAWAII-2 project, funded jointly by the IfA through the AEOS spectrograph contract from the Air Force Research Lab, by the Subaru telescope, and by ESO. While most basic design features of this device were copied from the HAWAII-1 devices, the HAWAII-2 demonstrated, for the first time, the feasibility of fabricating physically large multiplexers that exceed the reticle size of the stepper-aligners used for the silicon lithography. 


\section{Mosaic Arrays for NGST}

The latest round of infrared array developments are funded by NASA for the Next Generation Space Telescope (NGST) project. Competing for the contract to build the flight detector arrays for NGST are Rockwell Scientific, with the UH IfA as their academic partner, and the Raytheon Infrared Operations (formerly SBRC) with the U. of Rochester as their partner. The goal of these two competitive development projects is to demonstrate abuttable $2 \mathrm{~K} \times 2 \mathrm{~K}$ building blocks that can be assembled into the larger mosaic focal planes $(4 \mathrm{~K} \times 4 \mathrm{~K})$ needed for the NGST instruments. Both development projects work on detector material with a cutoff wavelength of $\approx 5 \mu \mathrm{m}$; Raytheon uses InSb while Rockwell will use $5 \mu \mathrm{m} \mathrm{MBE} \mathrm{HgCdTe} \mathrm{material,} \mathrm{that} \mathrm{has} \mathrm{been} \mathrm{demonstrated} \mathrm{to} \mathrm{at} \mathrm{least} \mathrm{match} \mathrm{the}$ performance of InSb (Hall et al. 2000).

NGST as a passively cooled $6 \mathrm{~m}$ class telescope in space is limited by the background of the zodiacal light and requires detector arrays of much better performance than ground-based imaging. For NGST spectroscopy, the performance will be detector limited under any realistic projection of detector performance, so the goal of these development projects are detector arrays of the best possible performance.

Compared to the conservative design of the HAWAII-2 arrays, Rockwell has implemented several substantial improvements in the design of the HAWAII$2 \mathrm{RG}$, their contender in this competition. The " $\mathrm{R}$ " in the device name stands for reference pixels, designed to give a stable reference signal independent of photon flux or detector dark current that in all other aspects mimics the electrical properties of detector elements. The "G" indicates a guide mode, a hardware subarray readout mode that allows to frequently read a small sub-array of the science array for guiding or wavefront-sensing purposes.

\section{Outlook}

The evolution of infrared arrays in the 1990 has led to their widespread use in astronomy. Still, today's infrared arrays leave room for improvement.

Quantum efficiency: Thinned, AR coated InSb typically achieves very high quantum efficiency, but PACE-1 HgCdTe typically exhibits somewhat lower q.e. $(60 \%)$ with a tendency to fall off further at short wavelengths. It is expected that the new generation of MBE deposited $\mathrm{HgCdTe}$, in particular on the latticematching CdZnTe substrate, will result in fewer defects in the material and better q.e..

Dark Current: Dark currents as low as $0.01 \mathrm{e} / \mathrm{s}$ have been achieved with $2.5 \% \mu \mathrm{m}$ PACE-1 HgCdTe. InSb dark currents are typically higher $(\approx 0.1 \mathrm{e} / \mathrm{s})$, which is not surprising for a longer wavelength material. Dark current is largely due to crystal lattice defects, and the new generation of MBE grown $\mathrm{HgCdTe}$ devices is achieving dark currents of $0.01 \mathrm{e} / \mathrm{s}$ for $5 \mu \mathrm{m}$ material.

The Raytheon Infrared Operations (RIO) is also developing new, abuttable $2 \mathrm{~K} \times 2 \mathrm{~K}$ devices specifically for ground-based operation. The ORION devices, developed in collaboration with NOAO, uses InSb as detector material, while the VIRGO devices will use LPE grown $\mathrm{HgCdTe}$ on Si substrates. 
Contrary to the situation in the recent past where Rockwell served the market for $2.5 \mu \mathrm{m}$ cutoff material with PACE-I $\mathrm{HgCdTe}$ while Raytheon provided InSb detectors with sensitivity out to $5.5 \mu \mathrm{m}$, we will soon see both manufacturers offer devices both for the $2.5 \mu \mathrm{m}$ and $5.5 \mu \mathrm{m}$ instruments.

All the most recent infrared array development projects are designing the devices to be abuttable at least in two directions. Most devices offer 3-side abuttability with close spacing and abuttability on the forth side with somewhat larger gaps. This, in principle, opens the way for focal plane mosaics of unlimited size. However, the high cost of infrared arrays, about one order of magnitude above that of optical CCDs, will, in practice, limit the size of available focal plane mosaics.

Another issue is the design of mosaic array controller and data acquisition systems. For large mosaics, the conventional approach of multi-channel controllers reaches practical limits in the number of wires required, the complexity and cost of these systems, and power consumption in the immediate vicinity of the infrared camera. Rockwell's development of an "application specific integrated circuit" (ASIC) dedicated to the control and data acquisition of HAWAII-2RG devices offers the intriguing possibility of simplifying infrared camera design significantly.

\section{Summary}

The HAWAII-2 arrays are now being implemented in the latest generation of instruments for the near-infrared. Although they were not designed to be closely abuttable, they are being used for the UKIRT WFC project of a wide field infrared survey camera.

The new generation of abuttable large-format infrared arrays will lead to wide-field survey cameras with fields of view of a good fraction of a square degree. Combined with the allocation of substantial fractions of the available time on some $4 \mathrm{~m}$ class telescopes to infrared survey work, large areas of the sky will be searched to depths vastly exceeding those of the 2MASS all-sky survey. Extremely sensitive and efficient instruments for follow-up work are being designed, in particular the new wide-field multi-slit spectrographs currently on the drawing boards.

\section{References}

Hall, D. N. B., Hodapp, K. W., Goldsmith, D. L., Cabelli, C., Haas, A. K., Kozlowski, L. J., \& Vural, K. 2000, SPIE 4008, 1268

Hodapp, K.-W., Hora, J. L., Hall, D. N. B., Cowie, L. L., Metzger, M., Irwin, E., Vural, K., Kozlowski, L. J., Cabelli, S. A., Chen, C. Y., Cooper, D. E., Bostrup, G. L., Bailey, R. B., \& Kleinhans, W. E. 1996, New Astronomy 1,117

Hoffman, A. W., Ando, K. J., Estrada, A. D., Garnett, J. D., Lum, N. A., Love, P. J., Rosbeck, J. P., Sparkman, K. P., Fowler, A. M., Pipher, J., \& Forrest, W., SPIE 3354, 24 